\title{
Synthesis of Carbon Dots with Tunable Luminescence
}

\author{
Smagulova SA*, Egorova MN, Tomskaya AE and Kapitonov AN \\ M.K. Ammosov North-Eastern Federal University, Yakutsk, Russia
}

\begin{abstract}
Synthesis of carbon dots carried out by the method of hydrothermal treatment of carbon precursors, such as glucose, citric acid and soot from birch bark in aqueous ammonia. The lateral dimensions distribution of the carbon dots was measured, from which the average dimensions were determined, which are in the range $10-12 \mathrm{~nm}$ in the case of glucose, citric acid and 20-22 nm in the case of soot.

Water suspensions with carbon dots from both glucose and citric acid show strong absorption in the region from 300 to $500 \mathrm{~nm}$, while carbon dots synthesized from soot have strong absorption in the ultraviolet region but are transparent in the visible region. The infrared absorption spectra were measured, which showed the presence of oxygen groups on the surface of synthesized carbon dots. Investigation of luminescence spectra have shown that carbon dots synthesized from glucose and soot luminesce equally in the violet region of the spectrum and the wavelength of the radiation is being dependent on the excitation wavelength. The luminescence intensity depends on the carbon dots dimensions and on the presence of oxygen groups on their surface. Luminescent carbon dots synthesized from glucose, citric acid and soot are ecological, biocompatible and have great prospects for their application in the field of biology and medicine.
\end{abstract}

Keywords: Hydrothermal synthesis; Carbon dots; Luminescence of carbon dots; Glucose; Citric acid; Soot; Nanotechnology

\section{Introduction}

In recent years, there is an increasing interest in the synthesis and study of quantum dots. Their main property is luminescence in the visible region of the spectrum. Among them, the most studied are semiconductor quantum dots, which have a high quantum yield, various unique optical and electrical properties. They are of the greatest interest in the field of bio-imaging and biomedicine, although their widespread use is severely limited in this area due to their several drawbacks, such as high toxicity, low biocompatibility, high cost and low chemical inertness. They show poor solubility/ bioavailability in aqueous solution [1]. Against this background, the synthesis of quantum dots based on carbon is becoming more urgent. Carbon dots (CDs) are a class of zero-dimensional carbon structures that contain $\mathrm{sp}^{2}$ carbon bonds and various functional groups. They are superior to conventional semiconductor quantum dots in terms of low toxicity, high biocompatibility, good chemical inertness and solubility [2]. They can also work as effective carriers of drugs because of their small size and biocompatibility [3]. In addition, their excellent photo- and electro-catalytic characteristics also allow application in the field of energy saving and environmental protection [4,5]. CDs demonstrate unique photoluminescent (PL) properties, which depend on several factors. First, luminescence can depend on the particle size and uniformity of the particle size distribution. Secondly, it depends on the surface passivating compounds, which affect not only luminescence, but also the radiation intensity. Thirdly, luminescence depends on the functionalization of the surface by organic molecules, which also contributes to tuning the wavelength range of the radiation. The possibility of adjusting the CDs photoluminescence parameters (wavelength, radiation intensity, quantum yield) with simple changes in the synthesis conditions makes CDs a promising material in various fields, depending on the necessary conditions for photoluminescence [6].

\section{Methods of Synthesis and Study of Carbon Dots}

The following reagents were used in hydrothermal synthesis: glucose $\left(\mathrm{C}_{6} \mathrm{H}_{12} \mathrm{O}_{6}\right.$, chemical purity, Ruskhim, Russia,), citric acid
$\left(\mathrm{C}_{6} \mathrm{H}_{8} \mathrm{O}_{7}\right.$, chemical purity, Chip-I-Dip, Russia), deionized water (DI), aqueous ammonia $\left(\mathrm{NH}_{3}\right.$, special purity $25.6 \%$, Vostokreaktiv, Russia). The following equipment was used for the synthesis process: polytetrafluoroethylene autoclave (TOPTION Laboratory Store, China), universal drying oven (SHSP-0.25-60-C, Russia), track membranes $(100 \mathrm{~nm})$, dialysis bag with pore size $3.5 \mathrm{kDa}$ (AXIOMBio, Russia), and magnetic stirrer.

The sequence of synthesis operations with a specific example of glucose is as follows: $2 \mathrm{~g}$ glucose was dissolved in $15 \mathrm{ml}$ of DI water, and then $6 \mathrm{ml}$ of aqueous ammonia (25\%) was added. The resulting solution was placed in a $50 \mathrm{ml}$ polytetrafluoroethylene autoclave. Hydrothermal synthesis was carried out in a drying oven at $180^{\circ} \mathrm{C}$ (and 1 hour) with a time variation of 10 minutes, 30 minutes, 1 hour, 4 hours, 8 hours, 10 hours, 18.5 hours. After the hydrothermal treatment, the suspension becomes brown, while the initial water solution with carbon precursors was transparent. The resulting brown suspension was dialyzed for 12 hours. After dialysis, the solution was filtered through a track membrane with pore sizes of $100 \mathrm{~nm}$. Dialysis bag solution was used in order to characterize the properties of the suspension with carbon dots. CDs from citric acid were synthesized similarly.

Soot as the precursor of CDs is a black powder obtained after pyrolysis of the birch bark. CDs from soot were prepared by the hydrothermal method too. To obtain $\mathrm{N}$-doped CDs from soot, $6 \mathrm{ml}$ of aqueous ammonia solution (25\%) and $15 \mathrm{ml}$ of DI water were added in $1 \mathrm{~g}$ of birch bark black. To obtain undoped CDs from soot, $15 \mathrm{ml}$ of DI

*Corresponding author: Smagulova SA, M.K. Ammosov North-Eastern Federal University, Yakutsk, Russia, Tel: +7 (4112) 496615; E-mail: smagulova@mail.ru

Received September 12, 2017; Accepted September 25, 2017; Published October 08, 2017

Citation: Smagulova SA, Egorova MN, Tomskaya AE, Kapitonov AN (2017) Synthesis of Carbon Dots with Tunable Luminescence. J Material Sci Eng 6: 376 doi: 10.4172/2169-0022.1000376

Copyright: @ 2017 Smagulova SA, et al. This is an open-access article distributed under the terms of the Creative Commons Attribution License, which permits unrestricted use, distribution, and reproduction in any medium, provided the original author and source are credited. 
water and $1 \mathrm{~g}$ of soot were mixed. The solutions were transferred to a poly-tetra-fluoro-ethylene autoclave, which was heated in a universal oven for 1, 2 and 4 hours. The resulting pale yellow solutions were also dialyzed for 12 hours and filtered through a $100 \mathrm{~nm}$ track membrane.

The luminescent properties of the suspensions were investigated using a Perkin Elmer LS 50B spectrometer (USA). The suspension was applied drop-wise to an oxidized silicon substrate study the characteristics of the carbon dots with a scanning electron microscope (JEOL JSM-7800F, Japan) and to measure FT-IR spectrum (Varian 7000 FT-IR, USA).

\section{Experimental Results}

In daylight, the suspensions of carbon dots synthesized from glucose (Figure 1a, upper) and from citric acid (Figure 1b, upper) are colored brown. When these suspensions were illuminated with an ultraviolet ray with a wavelength of $325 \mathrm{~nm}$, CDs from glucose glowed greenish light, while CDs from citric acid showed a blue fluorescence. Suspensions of CDs synthesized from soot are transparent (Figure 1c, upper), and their luminescence under the ultraviolet ray is green (Figure 1c, bottom).

The study of the obtained CDs by the scanning electron microscopy (SEM) method made it possible to determine their lateral dimensions. Figure 2a shows SEM images of carbon dots synthesized from glucose, in Figure 3 - from citric acid, in Figure 4 - from soot.

With synthesis duration of 1 hour, the average lateral dimensions of CDs from glucose are $11 \mathrm{~nm}$, from citric acid are $13 \mathrm{~nm}$, and from soot are $22 \mathrm{~nm}$ (Figures 2b, 3b and 4b). Elemental analysis showed that carbon dots synthesized from glucose consist of carbon - $44.1 \%$ (atomic), oxygen $48 \%$, nitrogen $7.8 \%$, whereas for CDs from soot as follows: $25.3 \%$, oxygen $-55.8 \%$, nitrogen $-19 \%$.

Figure 5 shows FT-IR spectrum of CDs synthesized from glucose and citric acid. FT-IR spectrum showed a difference in the content of oxygen groups for CDs synthesized from glucose and citric acid. In the case of glucose, the maximum concentration of oxygen groups on the surface of CDs is observed at 10 minutes of synthesis, and their concentration decreases sharply with a further increase in the synthesis time.

Figure 6 shows the FT-IR spectrum of CDs synthesized from soot, depending on the addition of ammonia to the initial solution. The addition of ammonia has virtually no effect on the FT-IR spectrum form, and conversely, the duration of synthesis strongly affects the FTIR spectrum of CDs. UV-VIS absorption spectrum for CDs synthesized from glucose are shown in Figure 7, depending on the synthesis time.

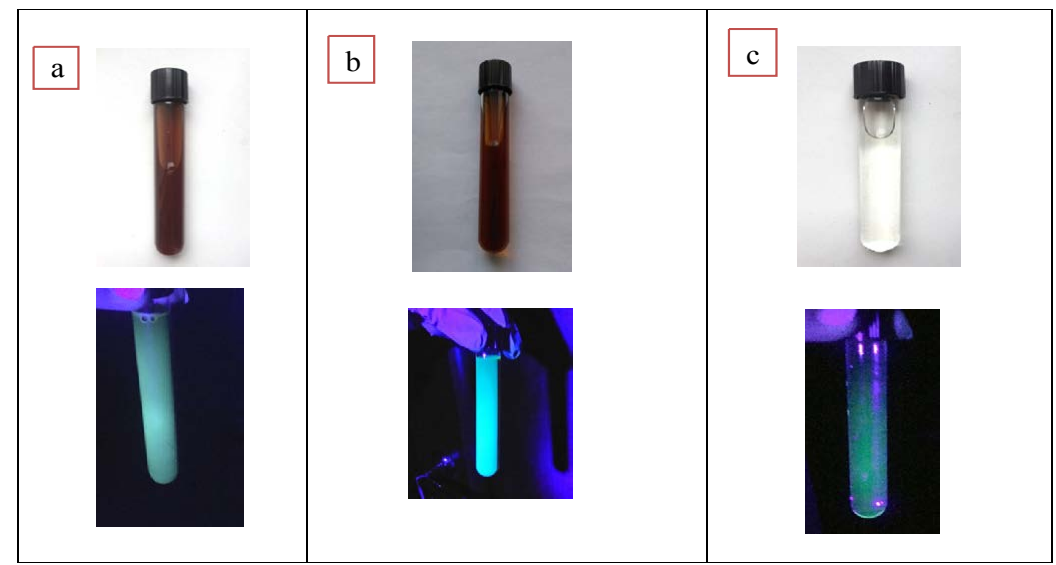

Figure 1: Solutions of CDs from a) glucose, b) citric acid; c) birch soot after hydrothermal treatment under daylight (upper) and under UV light ( $\lambda=325$ nm) (bottom image).
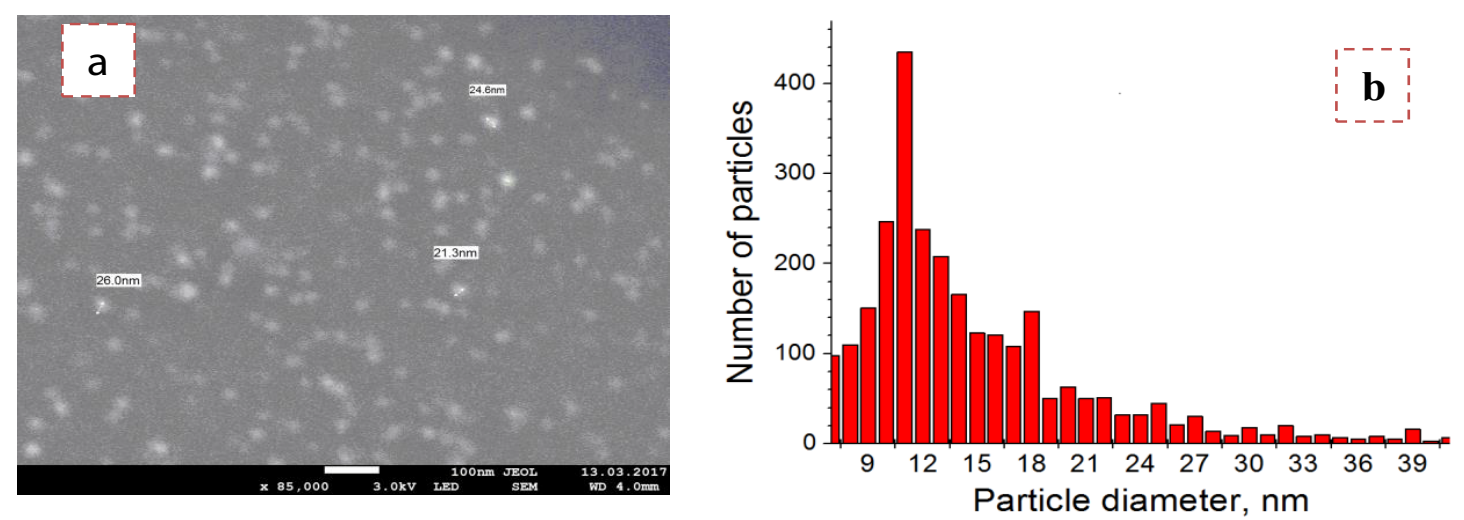

Figure 2: a) SEM image and b) distribution of lateral dimensions of CDs from glucose on a silicon oxide surface. 

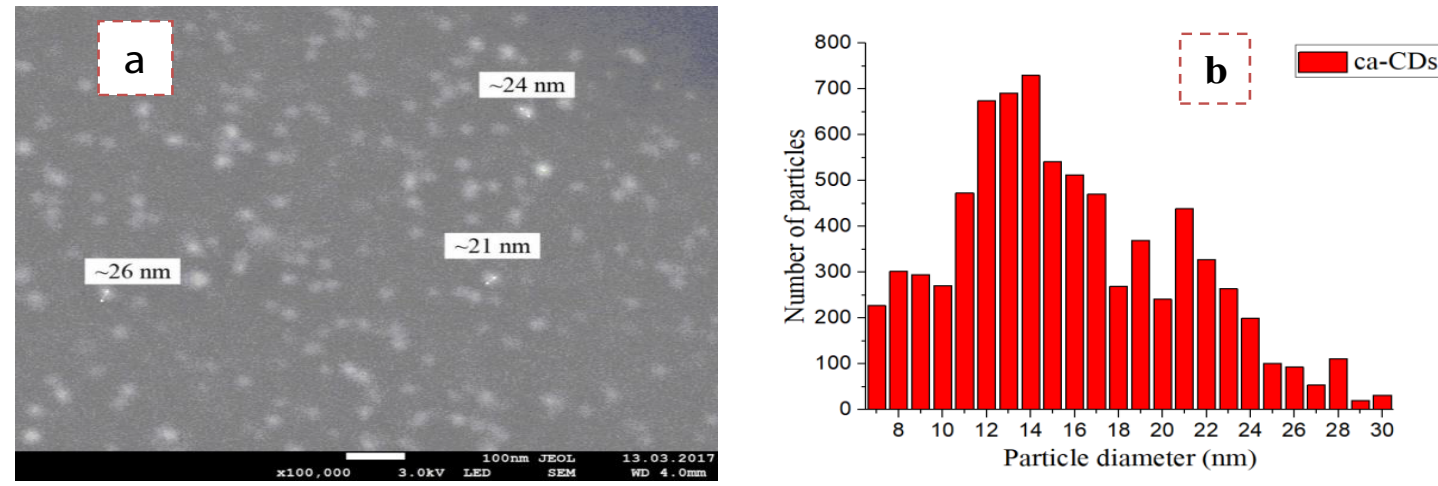

Figure 3: a) SEM image and b) distribution of lateral dimensions of CDs from citric acid on a silicon oxide surface.
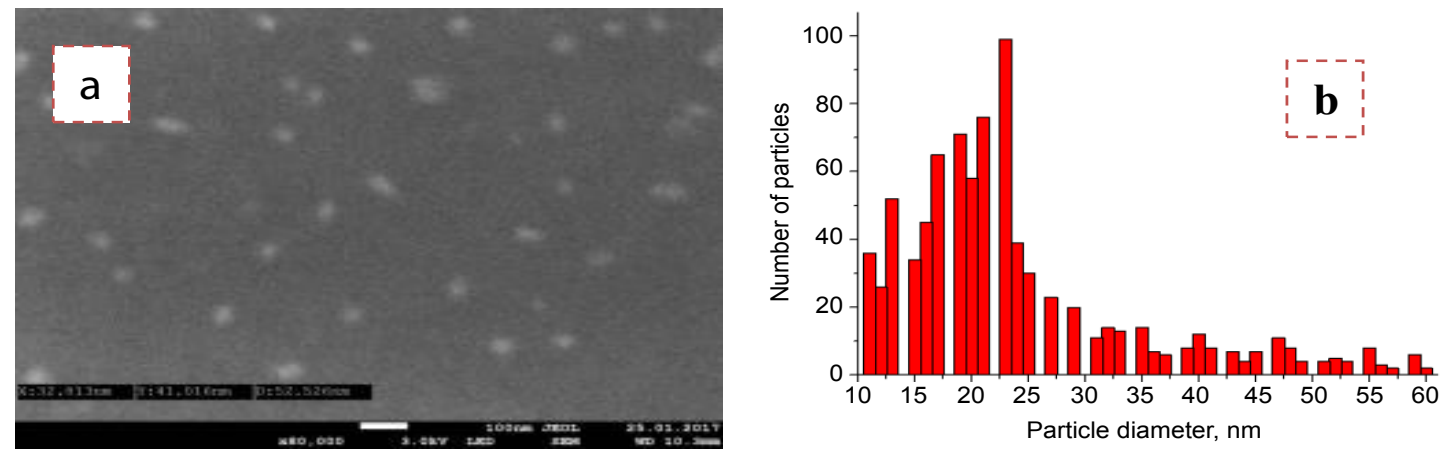

Figure 4: a) SEM image and b) distribution of lateral dimensions of CDs from birch soot on a silicon oxide surface.

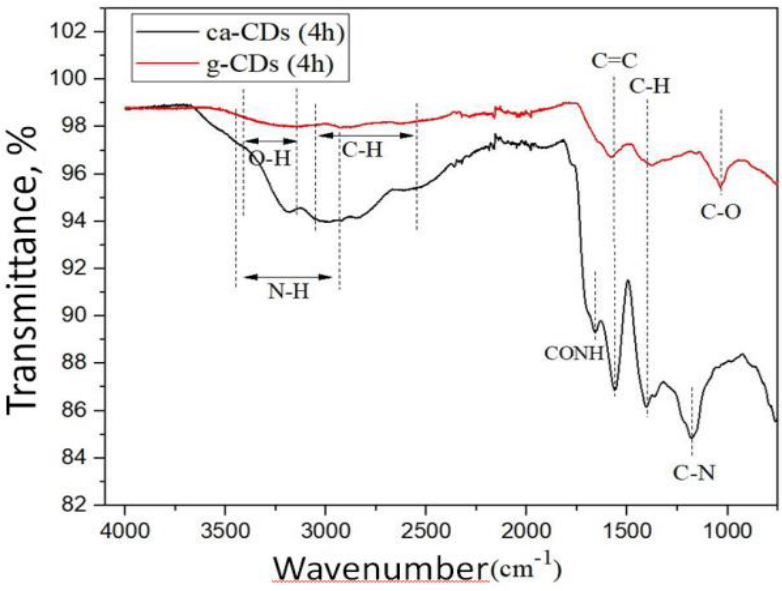

Figure 5: FT-IR spectrum of CDs. Black curve for CDs from citric acid (4 h), red curve for CDs from glucose $(4 \mathrm{~h})$.

The maximum absorption intensity is observed in the wavelength range from $380 \mathrm{~nm}$ to $440 \mathrm{~nm}$. The general form of the absorption spectrum slightly depends on the time of hydrothermal synthesis. Figure 8 shows UV-VIS absorption spectrum of CDs synthesized from soot with addition of ammonia to initial solution and without addition.

One can see in Figure 8 that the addition of ammonia significantly affects the UV-VIS absorption spectrum form. Absorption peaks at 205 $\mathrm{nm}, 220 \mathrm{~nm}, 270 \mathrm{~nm}$ are clearly seen in the case with nitrogen (a) while

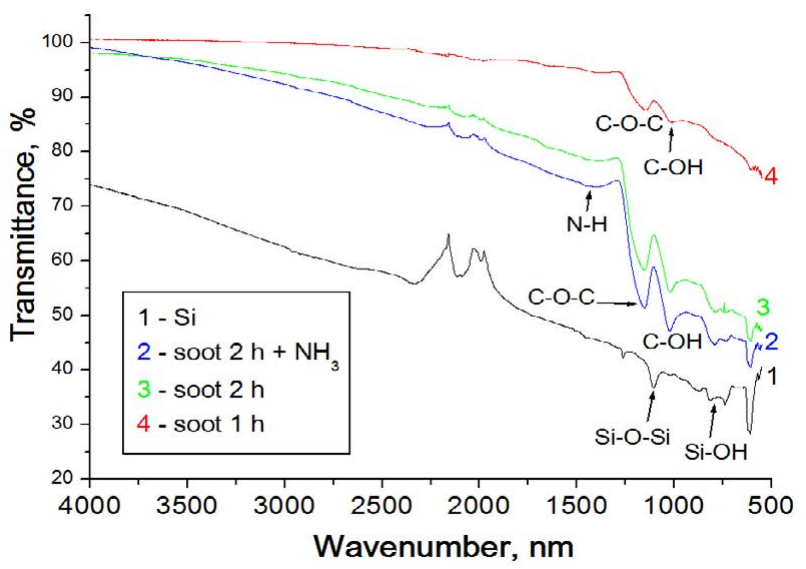

Figure 6: FT-IR spectrum: 1 - $\mathrm{SiO}_{2}$ substrate, 2 - soot $2 \mathrm{~h}$ with $\mathrm{NH}_{3}, 3$ - soot 2 h without $\mathrm{NH}_{3}, 4$ - soot 1 hour with $\mathrm{NH}_{3}$.

a continuous absorption curve is observed in the wavelength range from $200 \mathrm{~nm}$ to $300 \mathrm{~nm}$ without nitrogen (b).

Figure 9 shows the photoluminescence (PL) spectrum of CDs synthesized a) from glucose and b) from citric acid at different excitation lengths. For CDs from glucose, the luminescence peaks are shifted to the long-wavelength region by approximately $\Delta 70 \mathrm{~nm}$ with a change in the excitation wavelength from $300 \mathrm{~nm}$ to $400 \mathrm{~nm}$. For CDs from citric acid this displacement is practically not observed. 


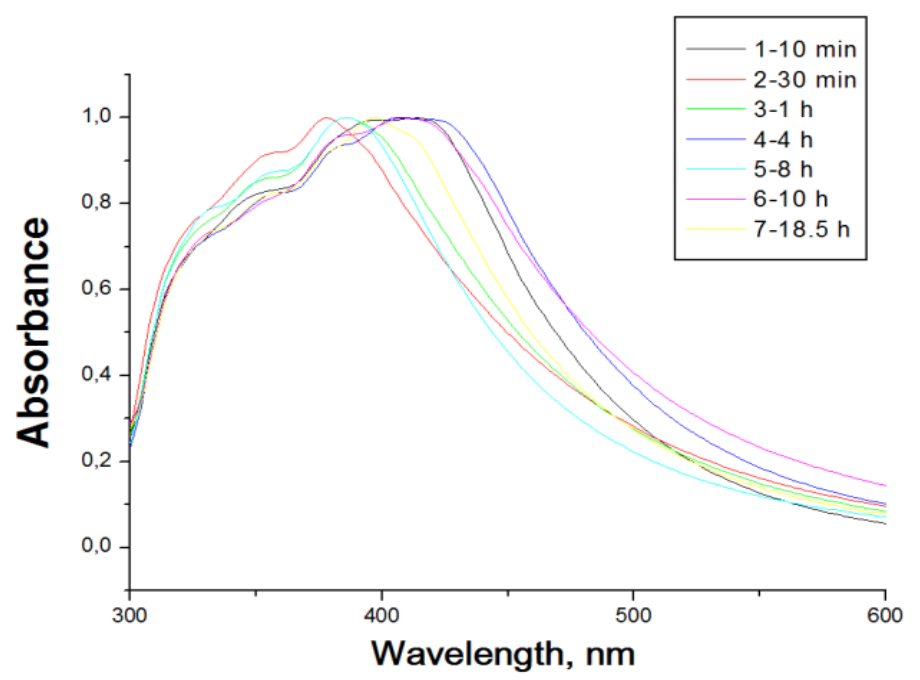

Figure 7: UV-VIS absorption spectrum of CDs from glucose after hydrothermal treatment for: 1-10 min, 2-30 min, 3-1 h, 4-4 h, 5-8 h, 6-10 h, 7-18.5 h.
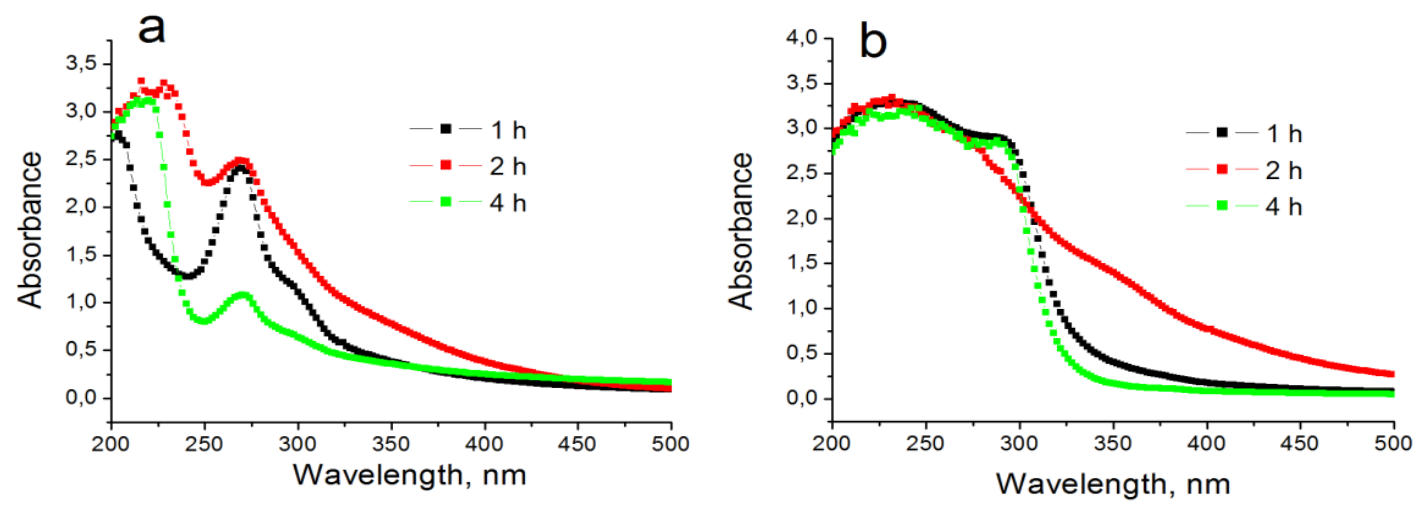

Figure 8: UV-VIS absorption spectrum solutions of CDs from soot: a) with $\mathrm{NH}_{3}$, b) without $\mathrm{NH}_{3}$ after hydrothermal treatment for: 1-1 h, 2-2 $\mathrm{h}, 3-4 \mathrm{~h}$.
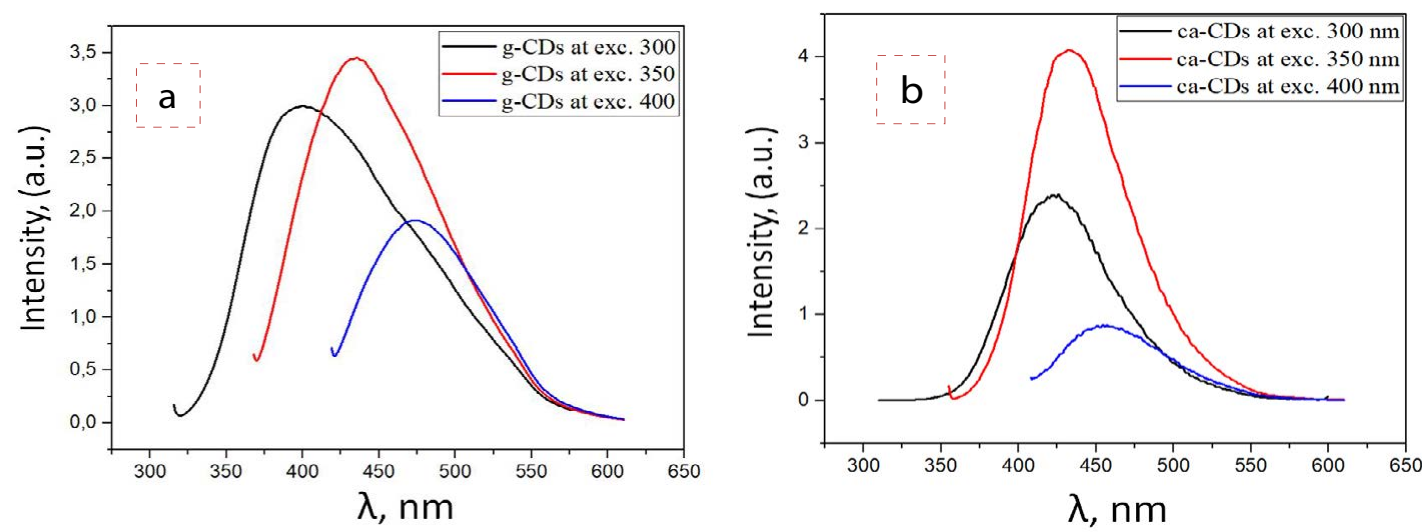

Figure 9: PL spectrum of CDs synthesized a) from glucose, b) from citric acid at different excitation wavelengths $(300,350,400 \mathrm{~nm})$.

A comparison made for the PL spectrum of CDs synthesized from glucose and citric acid is shown in Figure 10a. It is clearly that the luminescence peaks approximately shifted by $\Delta 50 \mathrm{~nm}$. Luminescence spectra for CDs synthesized from soot are presented in Figure 10b.
This figure shows that the luminescence peaks coincide for CDs from glucose and soot. When excited at a wavelength of $300 \mathrm{~nm}$, the luminescence intensity of CDs from soot is 15 times greater than for CDs from glucose. 

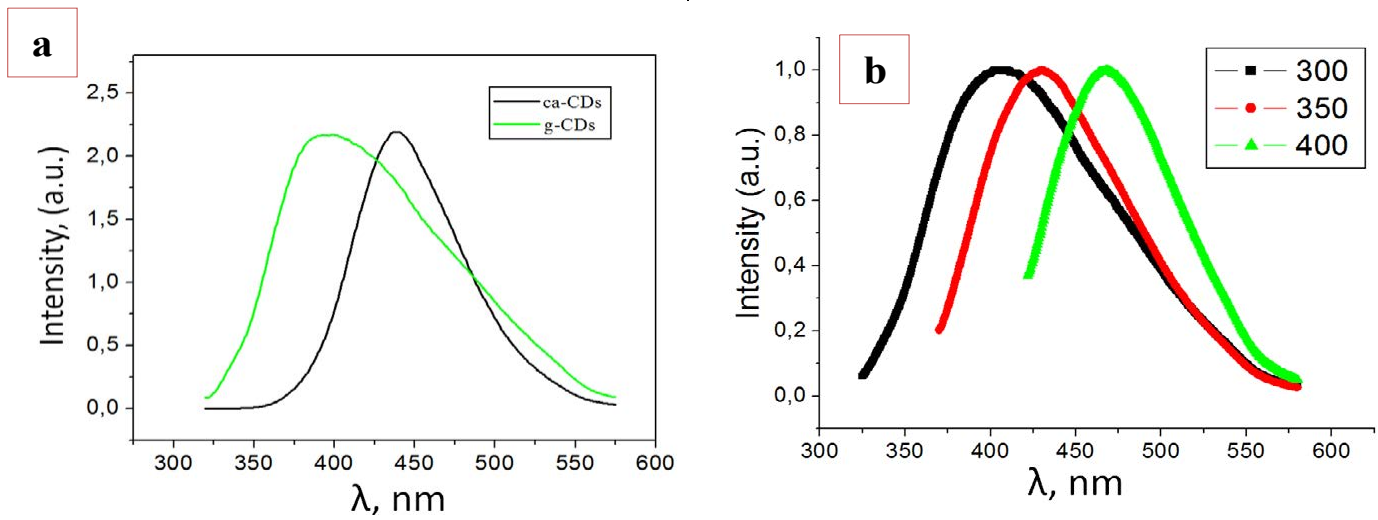

Figure 10: a) Comparison of the PL spectrum of CDs synthesized from glucose and citric acid; b) normalized PL spectrum of CDs from soot at different excitation wavelengths $(300,350,400 \mathrm{~nm})$.

\section{Discussion}

The basis of hydrothermal synthesis is the high solubility of substances in water at high pressure and temperature and the possibility of subsequent crystallization of the dissolved material from the liquid phase. High temperature of water plays an important role in the synthesis, because it creates an increased vapor pressure, the properties of the reagents vary (solubility, diffusion rate, reactivity), and water structure differs from that at room temperature. The control of vapor pressure, temperature and reaction time provides a great potential for the synthesis of high-quality CDs.

In our experiments, a temperature range of $180^{\circ} \mathrm{C}$ was chosen for hydrothermal synthesis of CDs as optimal for self-assembly of CDs from atoms and molecules in the initial suspension. The lateral size distribution of CDs from glucose and citric acid is close to the Gaussian distribution, and has a maximum at about $11 \mathrm{~nm}$ for glucose and 13 $\mathrm{nm}$ for citric acid for a synthesis time of 1 hour. For CDs in soot, the size distribution is asymmetric and the average size of the CDs is larger than in the first case and is within $22 \mathrm{~nm}$. Probably it is because in the case of soot in suspension, there are quite a lot of free carbon atoms that actively participate in the formation of the CDs nucleus. For CDs from glucose, we assume that the concentration of free carbon during synthesis is low, since the decay of glucose molecules is complex and multistage. It was found that the duration of synthesis increases the size of CDs, for example, for CDs synthesized from glucose, an increase in the synthesis time to 4 hours leads to an average size of $15 \mathrm{~nm}$.

FT-IR spectrum (Figure 5) showed that the carbon dots synthesized from glucose have a number of absorption centers, mainly associated with functional groups. After hydrothermal treatment glucose is dehydrated to form a $\mathrm{C}=\mathrm{C}$ bond, which is an elementary unit of synthesized CDs. The absorption peak at $1641 \mathrm{~cm}^{-1}$ is due to $\mathrm{C}=\mathrm{C}$ stretching. A wide peak at $3392 \mathrm{~cm}^{-1}$ indicates the presence of $\mathrm{O}-\mathrm{H}$. Absorption at $1027 \mathrm{~cm}^{-1}$ refers to valence vibrations of $\mathrm{C}-\mathrm{OH}$; the absorption at $2927 \mathrm{~cm}^{-1}$ refers to the vibrations of $\mathrm{C}-\mathrm{H}$, and the absorption at $1380 \mathrm{~cm}^{-1}$ indicates the presence of an $\mathrm{N}-\mathrm{H}$ bond (Figure 5). In addition to the vibrations of $\mathrm{C}-\mathrm{OH}, \mathrm{C}-\mathrm{O}-\mathrm{C}$, and $\mathrm{C}-\mathrm{H}$ bonds, the valence vibrations $\mathrm{C}=\mathrm{O}\left(\sim 1610 \mathrm{~cm}^{-1}\right)$ were also observed, in combination with asymmetric and symmetric valence vibrations of C-O-C $\left(\sim 1300 \mathrm{~cm}^{-1}\right.$ and $\left.\sim 1200 \mathrm{~cm}^{-1}\right)$ in the carboxyl group. It may be related to oxidation of $\mathrm{C}-\mathrm{OH}$ bonds by residual or decomposed oxygen atoms under hydrothermal treatment conditions [7-9]. The functional groups found on the surface of the CDs act as a passivating layer.
This layer facilitates the solubility of CDs in water, and enhances the properties of photoluminescence [10]. The presence of a large number of residual hydroxyl groups improves the hydrophilicity and stability of carbon dots in polar solvents [6,10]. These hydrophilic groups not only give an idea of the luminescence mechanisms, but also contribute to the harmlessness of the product, which makes it possible to use them in biochemical studies. As shown by the FT-IR spectrum for CDs from glucose (Figure 5), the concentration of functional groups on the surface of CDs is mainly affected by the duration of the synthesis process. As the synthesis time increases, the intensity of the absorption bands decreases, which is explained by the decrease in the number of functional groups on the CDs surface as a result of intramolecular changes in the composition of functional groups under hydrothermal treatment.

UV-VIS absorption spectrum of CDs synthesized from glucose (Figure 7) and soot (Figure 8) strongly differ from each other. This fact apparently is an argument for the qualitative composition and dimensions of CDs are very different. In the case of CDs from soot (Figure $8 \mathrm{a}$ and $8 \mathrm{~b}$ ), the presence of nitrogen atoms strongly affects the character of the absorption. Ammonia plays two roles in the growth of N-CDs. First, it acts as a catalyst for intra- and intermolecular reactions, and secondly, for the doping of carbon dots with nitrogen. It is possible that the presence of nitrogen atoms in the CDs nucleus, introducing certain deformation distortions, contributes to a better capture of functional groups on the CDs surface.

The peaks of the PL spectrum for CDs synthesized from glucose (Figure 9a) and soot (Figure 10b) coincide. They also shift equally to the long-wavelength region with a change in the excitation wavelength (350, 400, $450 \mathrm{~nm}$.). This suggests that the luminescence centers in both cases are the same functional groups on the CDs surface. It is known from the literature [11] that the "bare" carbon nucleus does not luminesce. The ratio of luminescence intensities of CDs synthesized from soot and glucose is non-monotonic: at a excitation wavelength of $300 \mathrm{~nm}$ it is 15 times larger (Figure 7), at $350 \mathrm{~nm}$ approximately of the same order, at $400 \mathrm{~nm}-1.7$ times larger. Such a dependence of the luminescence intensity can be explained by the low intensity of CDs absorption in the region from $300 \mathrm{~nm}$ to $400 \mathrm{~nm}$ (Figure 7).

\section{Conclusion}

Optimal conditions for hydrothermal synthesis of carbon dots from glucose, citric acid, soot were found: reaction time 3-4 hours, 
temperature range $180^{\circ} \mathrm{C}$; the average sizes of $\mathrm{CDs}$ were determined, which lie in the range of $11-13 \mathrm{~nm}$ for glucose, citric acid and $22 \mathrm{~nm}$ for soot. FT-IR is showed the presence of bonds: C - 44.1\%, O - 48.1\%, $\mathrm{N}-7.8 \%$ for glucose and C $-25.3 \%, \mathrm{O}-55.8 \%, \mathrm{~N}-18.9 \%$ for soot. In the IR region, absorption on the bonds is visible: $\mathrm{C}=\mathrm{C}, \mathrm{N}-\mathrm{H}, \mathrm{C}-\mathrm{OH}$, $\mathrm{C}-\mathrm{O}-\mathrm{C}, \mathrm{CONH}, \mathrm{C}-\mathrm{O}$. It has been established that the absorption time is affected by the time of hydrothermal synthesis. An aqueous suspension of CDs from glucose showed strong absorption in the region from 300 $\mathrm{nm}$ to $500 \mathrm{~nm}$, while CDs from soot have strong absorption in UV, but are transparent in the visible region. It is shown that the presence of nitrogen leads to the appearance of two peaks in the UV region: $260 \mathrm{~nm}$ and around $200 \mathrm{~nm}$. It is shown that CDs, synthesized from glucose, soot, citric acid have luminescence in the UV and blue regions: $380-470$ $\mathrm{nm}$. The intensity of luminescence is maximal in the case of glucose, 9 times greater than for $\mathrm{CDs}$ from soot at equal concentrations. In the $\mathrm{CDs}$ spectrum, the soot contains no absorption by $\mathrm{C}=\mathrm{C}, \mathrm{CONH}$. The sizes of CDs from glucose are less than the size of CDs in soot and in addition, the core of CDs from soot is amorphous. It is suggested that these three factors explain the difference in the 1 uminescence intensities. Hydrothermal synthesis of CDs from different carbon precursors has great prospects for obtaining luminescent centers with variable wavelength and intensity, suitable for development of the optoelectronic devices.

\section{Acknowledgements}

The results were obtained within the performing of the state task of the Ministry of Education and Science of Russia "Leading Researchers" No. 16.6824.2017/6.7.

\section{References}

1. Merian J, Gravier J, Navarro F, Texier I (2012) Fluorescent nanoprobes dedicated to in vivo imaging: from preclinical validations to clinical translation. Molecules 17: 5564-5591.

2. Dong Y, Pang H, Yang HB, Guo C, Shao J, et al. (2013) Carbon-Based Dots Co-doped with Nitrogen and Sulfur for High Quantum Yield and Excitation Independent Emission. Angew Chemi Int Ed 52: 7800-7804.

3. Dimos K (2016) Carbon quantum dots: Surface passivation and functionalization. Current Organic Chemistry 20: 682-695.

4. Tang L, Ji R, Cao X, Lin J, Jiang H, et al. (2012) Deep ultraviolet photoluminescence of water-soluble self-passivated graphene quantum dots. ACS nano 6: 5102-5110.

5. Wang X, Qu K, Xu B, Ren J, Qu X (2011) Microwave assisted one-step green synthesis of cell-permeable multicolor photoluminescent carbon dots without surface passivation reagents. Journal of Materials Chemistry 21: 2445-2450.

6. Eda G, Lin YY, Mattevi C, Yamaguchi H, Chen HA, et al. (2010) Blue photoluminescence from chemically derived graphene oxide. Advanced materials 22: 505-509.

7. Wu ZL, Zhang P, Gao MX, Liu CF, Wang W, et al. (2013) One-pot hydrothermal synthesis of highly luminescent nitrogen-doped amphoteric carbon dots for bioimaging from Bombyxmori silk-natural proteins. Journal of Materials Chemistry B 1: 2868-2873.

8. Du F, Jin X, Chen J, Hua Y, Cao M, et al. (2014) Nitrogen-doped carbon dots as multifunctional fluorescent probes. Journal of nanoparticle research 16: 2720.

9. Eliseev $A(2010)$ Functional nanomaterials M: Fizmatlit 456,

10. Zhu H, Wang X, Li Y, Wang Z, Yang F, et al. (2009) Microwave synthesis of fluorescent carbon nanoparticles with electrochemiluminescence properties. Chemical Communication 34: 5118-5120.

11. Sun YP, Zhou B, Lin Y, Wang W, Fernando KS, et al. (2006) Quantum-sized carbon dots for bright and colorful photoluminescence. Am Chem Soc 128: 7756-7757. 